\title{
DAMAGE QUANTIFICATION FOR CONCRETE STRUCTURES BY IMPROVED $b$-VALUE ANALYSIS OF AE
}

\author{
T. Shiotani ${ }^{1}$, X. Luo $^{2}$, H. Haya ${ }^{2} \&$ M. Ohtsu ${ }^{3}$ \\ ${ }^{1}$ Research Institute of Technology, Tobishima Corporation, 5472 Kimagase, Noda, Chiba 270-0222, Japan \\ ${ }^{2}$ Railway Technical Research Institute, 2-8-38 Hikari-machi, Kokubunji, Tokyo 185-0034, Japan \\ ${ }^{3}$ Graduate School of Engineering, Kumamoto University, 2-39-1 Kurokami, Kumamoto 860-8555, Japan
}

\begin{abstract}
Damage evaluation of concrete structures, in general, has been investigated visually. Except for seriously damaged structures, as a result, evaluation of moderately or intermediately damaged structures is usually difficult. Although prospective procedures using AE testing have already been proposed, the difference of monitoring condition in each application sometimes leads to non-quantitative results. Improved $b$-value is a slope of the peak amplitude distribution of AE signals. This value is known to be not influenced by the monitoring conditions, and to uniquely vary depending on the damage level. Accordingly, the improved $b$-value is applied to quantify the damage degree in damaged concrete piers due to an earthquake. Two sensor arrays with different damage levels are employed, and improved $b$-value based on the AE events located within the pier is obtained. As a result, the damage degree can be reasonably quantified by the improved $b$-value, which is promisingly employed as a damage index.
\end{abstract}

\section{INTRODUCTION}

By means of $\mathrm{AE}$ testing two indices have so far been used to inform the damage level. Felicity ratio (Fowler [1]) is one of those indices obtained from the stress level applied in comparison with the maximum stress level, and other is Calm ratio obtained from a ratio of accumulated number of $\mathrm{AE}$ activity during uploading to that during unloading. For the Felicity ratio, it is difficult to apply for in-situ monitoring since the maximum stress of which the materials have experienced is not readily estimated so that authors have proposed the RTRI instead (Luo et al. [2]). The RTRI is obtained on the basis of the maximum value during "a inspection period," and therefore the maximum value experienced has not been necessarily required in the calculation. Through the applications of those damage indices for in-situ concrete structures, however, several issues to be resolved still exist, for example, measurement of deformation is mandatory when obtaining the RTRI, moreover the deformation should exceed the minimal resolution of the value, otherwise it becomes difficult to obtain the onset of AE activity with respect to the deformation. Details discussion can be found in reference (Shiotani et al. [3,4]). Besides those indices, AE peak amplitudes are known to be closely related to the scale of fracture. Thus, the scale of peak amplitude might be larger with progress of fracture. However, it seems difficult only to pay attention to the peak amplitude. Because as fracture develops, an apparent 
mechanical property of the structure would change as well, in short, condition of media of which AE signals travel would be worse i.e., a high attenuation rate causes the fact that large scale of AE peak amplitude at the source results in small scale at the sensor. Thus, in the present paper the peak amplitudes are going to be studied as their distributions.

\section{PEAK AMPLITUDE DISTRIBUTION}

To analyze $\mathrm{AE}$ data acquired, a parameter analysis is widely and frequently employed. Among $\mathrm{AE}$ parameters, in particular, the $\mathrm{AE}$ peak-amplitude is considered a closely related parameter to the magnitude of fracture. Many researchers have thus studied $b$-value and $m$-value determined from a negative gradient of the peak-amplitude distribution. To apply the $b$-value analysis for fracture evaluation in slope failure, the method of calculating the $b$-value has been modified by incorporating statistical values of amplitude distribution (Shiotani et al. $[5,6])$. This is now referred to as improved $b$-value (I $b$-value) analysis.

\subsection{Improved b-value}

The range of AE amplitude in the improved $b$-value analysis is determined based on such statistical values as the mean $\mu$ and standard deviation $\sigma$. Setting the accumulated amplitude over $w_{1}$ and $w_{2}$, as $N$ $\left(w_{1}\right)$ and $N\left(w_{2}\right)$, Ib-value is given by,

$$
I b=\frac{\log _{10} N\left(w_{1}\right)-\log _{10} N\left(w_{2}\right)}{\left(\alpha_{1}+\alpha_{2}\right) \sigma},
$$

where $\alpha_{1}$ and $\alpha_{2}$ are constants.

The other modification in the $\mathrm{I} b$-value analysis over the conventional $b$-value is the number of $\mathrm{AE}$ event analyzed. The number of $\mathrm{AE}$ data points is formulated by,

$$
\int_{0}^{\infty} n(a) d a=\beta,
$$

where $n(\mathrm{a})$ is the number of $\mathrm{AE}$ hits (or events) at $d a$ and $\beta$ is the total number of $\mathrm{AE}$ hits (or events)
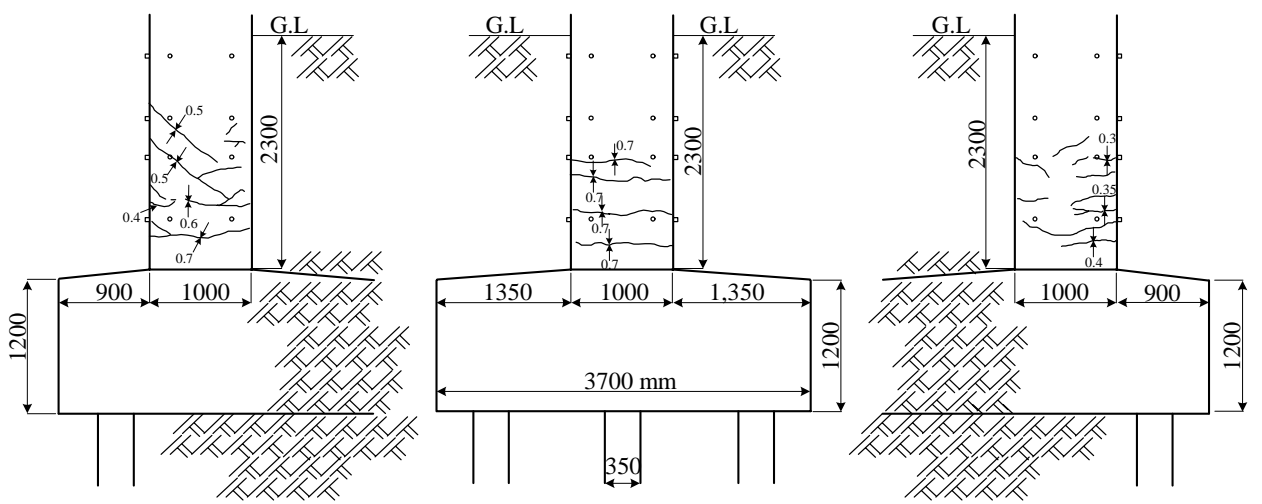

Figure 1: Crack condition of the RC pier, showing north, east, and south side from left to right. 


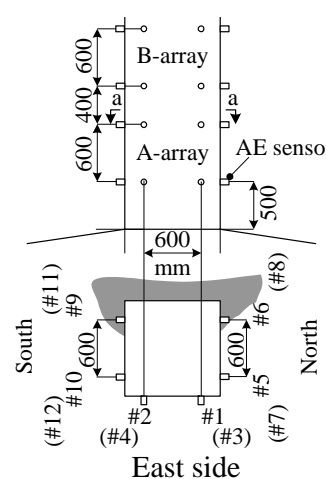

Figure 2: Two arrays of AE sensors.

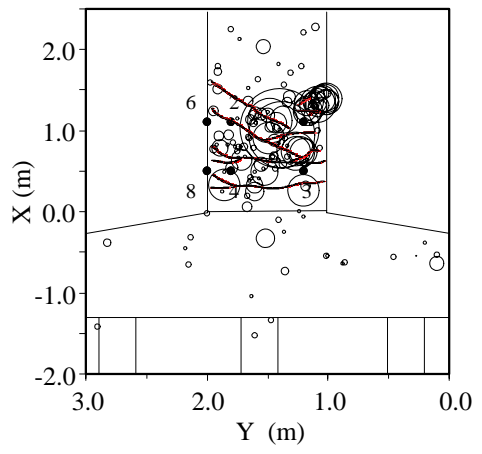

(a) A-array

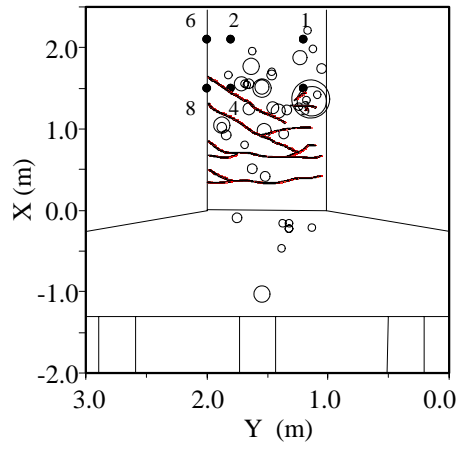

(b) B-array

data subjected to the $\mathrm{I} b$-value analysis. Any number over 50 is considered appropriate as $\beta$. In consideration of the problem in population parameters, $\beta$ from 50 to 100 would be an appropriate range to calculate the $\mathrm{I} b$-value. (Shiotani et al [5]). An appropriate number of $\beta$ in actual concrete structures are further studied in the session followed. When a comparison between the seismic $b$-value and $\mathrm{I} b$-value is made, the $\mathrm{I} b$-value should be multiplied by 20 .

\section{IN-SITU APPLICATION}

\subsection{Structures \& monitoring condition}

AE monitoring was conducted in a RC pier of a railway rigid-frame bridge. In the pier, notable cracks were not found above the ground but seriously damaged condition was readily found after ground excavation. The crack condition is shown as in Fig. 1 . In the north side, for example, a crack of $0.7 \mathrm{~mm}$ wide was found just above the footing and a diagonal crack, namely a shear type of crack, was observed at $1 \mathrm{~m}$ above the footing. Those cracks distributed up to $1.5 \mathrm{~m}$ above the footing. To know the $\mathrm{AE}$ activity not only for seriously damaged but intermediately damaged state, two arrays of $\mathrm{AE}$ sensors were employed i.e., A-array and B-array as shown in Fig. 2. A-array corresponds to seriously damaged state while B-array shows the intermediately damaged state. In each array, $12 \mathrm{AE}$ sensors of $60 \mathrm{kHz}$ resonance surrounds the pier. $\mathrm{AE}$ events induced by train passage were subsequently amplified by $40 \mathrm{~dB}$ at preamplifiers and fed to AE monitoring system (Mistras, Physical Acoustics Corp.) Both $\mathrm{AE}$ parameters and waveforms are recorded with the system.

\subsection{Results}

\subsection{1 $3 D$ AE sources}

AE sources induced by train passage are shown as in Fig. 3. 3D sources are all projected to the north sides plane, and the scale of the circle reflects the averaged peak amplitude of all hits contributing to the AE source/event. Fig. 3a was obtained from 10 times train passage, whereas Fig. $3 \mathrm{~b}$ was from 4 times train passage, thus the number of sources, except for the scale of $\mathrm{AE}$ sources, could not be discussed here. A-array shows a lot of AE sources generated actively around the observed cracks (see Fig. 3a), and the scale of AE sources distributed widely, namely from small to large magnitude. In 


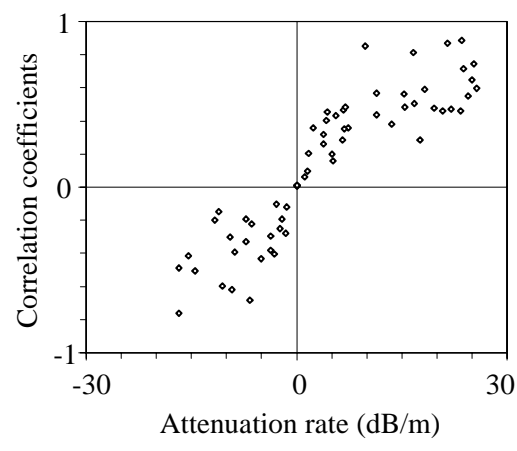

Figure 4: Attenuation rates.

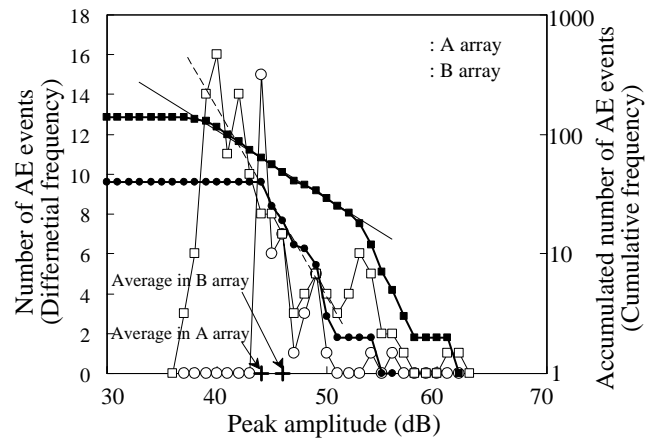

Figure 5: AE Peak amplitude distributions.

B-array, however, the large scale of AE sources as that in A-array could not be derived, and almost the identical scale of AE sources were presented.

\subsubsection{Peak amplitude at $A E$ sources}

Based on the AE sources obtained in A-array and locations of AE sensors, attenuation rates were calculated as in Fig. 4. The negative coefficients only show the attenuation provided that AE energy decays as a function of propagation distance, but positive coefficients were presented in the chart i.e., not only attenuation but an amplification characteristic was obtained. This implies that it is difficult to obtain attenuation rates in the materials of which cracks are randomly and intensively distributed. For example, in the case that a sensor was closely located to an AE source but a large number/scale of cracks was distributed in the vicinity of the sensor, resulting peak amplitude at the sensor would be smaller magnitude, while in the case that only a small number/scale of cracks was existed around the sensor, resulting peak amplitude at the sensor would be larger magnitude. This finding also suggests that in the structures of which heterogeneous crack-distribution from small to large scale are included, the peak amplitude at the source resulted in being difficult to calculate from obtained AE signals.

\subsubsection{Peak amplitude distributions}

AE peak amplitude distributions are exhibited as in Fig. 5. Open circles (B array) and open squares (A array) show the differential type of distribution and solid circles and solid squares show the cumulative type of distribution. In the figure, the averages of peak amplitudes in both arrays from all derived AE events are indicated with cross symbols as well. Again A-array corresponds to seriously damaged state while B-array shows the intermediately damaged state. The figure shows that the average of peak amplitude in B-array appeared larger value than that in A-array, suggesting that AE events generated from the intermediately damaged state had a larger value than seriously damaged state. Since, in general, the larger the scale of fracture becomes, the larger the AE peak amplitude has, the fact of the averages of peak amplitude does not suffice the general idea. In the differential type of distribution in B-array, AE events distributed narrowly around $45 \mathrm{~dB}$, and $\mathrm{AE}$ events of larger than $50 \mathrm{~dB}$ did not appear so much, whereas it distributed widely from small scale to large scale of amplitude (see around $40 \mathrm{~dB}$ and $55 \mathrm{~dB}$ ) in A-array. The difference of the differential distribution between both arrays resulted in thus the antithesis of general ideal. Solid plots show the cumulative types of distribution. In A-array, a descending curve with regard to the peak amplitude was linearly presented from 40 to $55 \mathrm{~dB}$, 

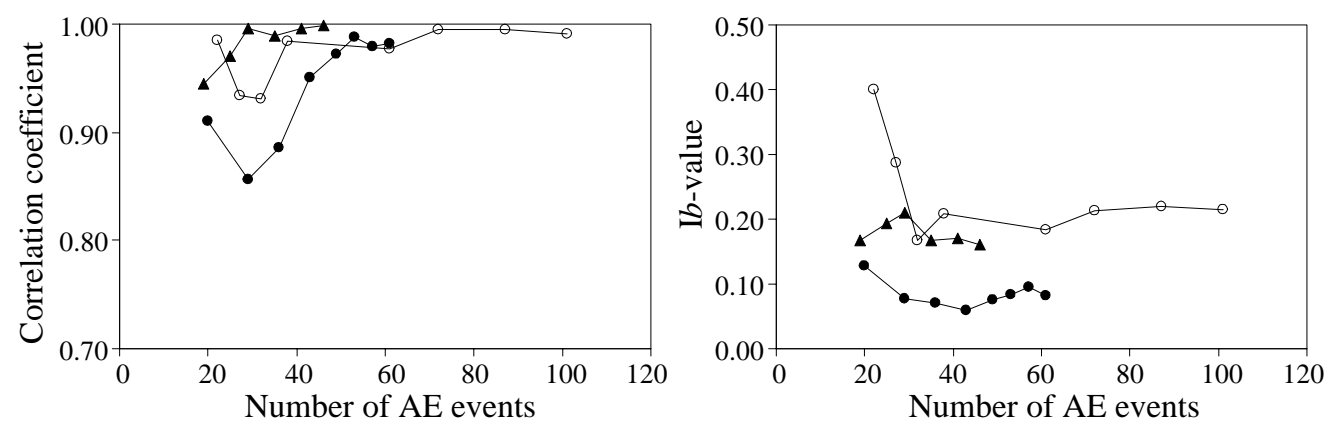

Figure 6: Correlation coefficients: left and improved $b$-value: right with the number of AE events.

where the gradient of the curve, when linear approximation is performed, are referred to as $b$-value. As in the same way, the cumulative distribution in B-array shows a decreasing trend as a function of peak amplitude, however, it can be readily found that the gradients would differ with the amplitude range employed. To resolve the difference in $b$-value depending on the range adopted, the improved $b$-value analysis ought to be performed (see eqn 1). The improved $b$-value analysis was conducted for both distributions of A-array and B-array. A solid line shows the approximated line of A-array and a dotted line indicates that of B-array. The gradient in A-array $(=0.05)$ appeared smaller value than that in B-array (= 0.1$)$, implying the improved $b$-value becomes smaller with damage levels.

\section{DISCUSSION}

\subsection{Required number of $A E$ data for the improved $b$-value analysis}

To obtain the reasonable number of $\mathrm{AE}$ events in the improved $b$-value analysis, correlation coefficients and resulted improved $b$-values are shown with respect to the number of AE events as in Fig. 6. Three different populations of AE events, namely 46 (triangle), 61 (solid circle) and 101 (open circle), are shown. The correlation coefficients become more than 0.98 when AE events more than 40 are obtained (see Fig. 6 left), correspondingly improved $b$-value shows consistent when more than 40 events (see Fig. 6 right). This implies that the reasonable number of $\mathrm{AE}$ events contributing to the improved $b$-value analysis would be more than 40 events, in short, it is difficult to quantify the damage with the improved $b$-value from the number less than 40 events.

\subsection{Improved b-value based on the results of one-dimensional source location}

The improved $b$-values shown above were derived on the basis of 3D source locations, and therefore 3D source location seems to be necessarily performed when obtaining the improved $b$-value. Suppose in-situ damage investigation is performed with $\mathrm{AE}$ monitoring, installation of a large number of $\mathrm{AE}$ sensors into the structures might be in practical since it would take much time and cost. Fig. 7 shows the cumulative distribution of $\mathrm{AE}$ events when one dimensional source location was conducted. Due to small numbers of AE events in B-array, the chart only shows the result of A-array. The cumulative distributions were resulted from one dimensional sensor array with: ch-9 and ch-11 (68), ch-2 and ch-4 
(42), and ch-5 and ch-7 (27) respectively, where the values in parentheses denote the $\mathrm{AE}$ events obtained. The resulting improved $b$-value from those three-combination of $\mathrm{AE}$ sensors, appeared smaller than 0.05 although the approximation becomes more difficult when the smaller number of events was derived. Conclusively, the improved $b$-value analysis is possibly performed using a small number of $\mathrm{AE}$ sensors, for example, two sensors at minimum, however, the number of $\mathrm{AE}$ events contributing to the improved $b$-value analysis should always to be considered, i.e., as shown in Fig. 6, the AE events smaller than 40 might cause unstable/ nonquantitative improved $b$-value, and the Fig. 7

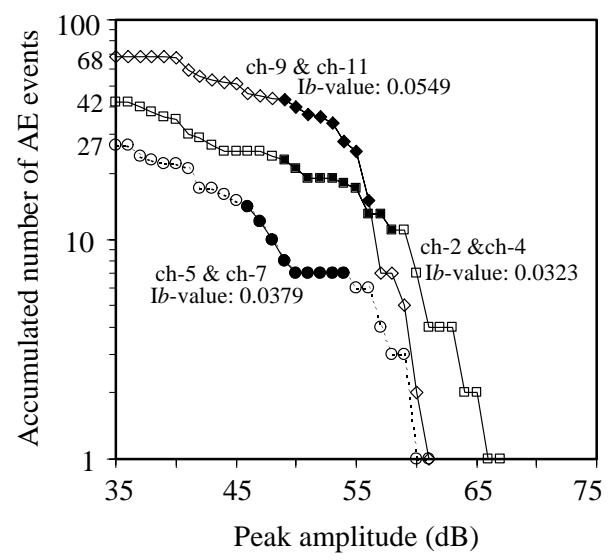

Figure 7: AE peak amplitude distributions in 1D source locations. accords well to the fact (see ch-5 \& ch-7).

\section{CONCLUSION}

To quantify the damage of concrete structures, the improved $b$-value analysis, obtained from the $\mathrm{AE}$ amplitude distributions, was applied for the in-situ RC piers damaged. Two damaged areas with different damage levels were subjected to the AE monitoring. The results showed that it was difficult to discuss damage levels by only using peak amplitudes. Since the improved $b$-value appeared different values depending on the damage levels, the damage degree could be reasonably quantified with the improved $b$-value. Even with a small number of $\mathrm{AE}$ sensors, the improved $b$-value analysis was possible so that it will be potentially employed as a damage index in practical investigations.

\section{Reference}

1) Fowler, T.J., Experience with acoustic emission monitoring of chemical process industry vessels, Progress in Acoustic Emission III, JSNDI: 150-162, 1986. 2) Luo, X., H. Haya, T. Inaba, T. Shiotani and Y. Nakanishi, Experimental study on evaluation of breakage in foundations using train-induced acoustic emission, Proc. Structural Engineering World Congress 2002: Paper No. T9-1-e-3, 2002, 3) Shiotani, T., Y. Nakanishi, X. Luo, H. Haya and T. Inaba, Damage evaluation for railway structures by means of acoustic emission, Proc in APCNDT 2003, Trans Tech Publications Ltd., Switzerland, in review. 4) Shiotani, T., Y. Nakanishi, X. Luo, H. Haya and T. Inaba, Evaluation of structural integrity in railway structures using train-induced acoustic emission, Structural Faults and Repair 2003, Engineering Technics Press, (CD-ROM), 2003. 5) Shiotani, T., K. Fujii, T. Aoki and K. Amou, Evaluation of progressive failure using AE sources and improved $b$-value on slope model test, Progress in Acoustic Emission VII, JSNDI: 529-534, 1994. 6) Shiotani, T. and M. Ohtsu, Prediction of slope failure based on AE activity, Acoustic Emission: Standards and Technology Update, ASTM STP 1353: 156-172, 1999. 